\title{
Efecto de la incorporación de ceniza volante y escoria de horno alto en el comportamiento electroquímico de concretos de cemento comercial
}

\author{
Oscar J. Gutiérrez-Junco ${ }^{\mathrm{a}, \varpi}$, Yaneth Pineda-Triana $^{\mathrm{b}}$, Enrique Vera-López ${ }^{\mathrm{b}}$ \\ ${ }^{a}$ Universidad Pedagógica y Tecnológica de Colombia, Facultad de Ingeniería, Avda. Central del Norte 39-115, Tunja, Colombia \\ ${ }^{b}$ Universidad Pedagógica y Tecnológica de Colombia, Instituto para la Investigación e Innovación en Ciencia y \\ Tecnología de Materiales (INCITEMA), Avda. Central del Norte 39-115, Tunja, Colombia \\ Autor para la correspondencia: oscarjavier.gutierrez@uptc.edu.co
}

Enviado: 4 Junio 2015; Aceptado: 4 Octubre 2015; Publicado on-Line: 12 N oviembre 2015

\begin{abstract}
RESUMEN: Este trabajo presenta los resultados de la evaluación de propiedades de pastas de cemento comercial (CPC), mezcladas con ceniza volante (FA) y escoria de alto horno (GBFS). Inicialmente un total de 30 combinaciones fueron evaluadas en términos de resistencia a la compresión para establecer las proporciones óptimas de las materias primas. Después de esto, cuatro mezclas optimizadas fueron caracterizadas durante el proceso de fraguado y endurecimiento. Se realizaron ensayos electroquímicos en cilindros de concreto elaborados con los cementantes y con una varilla de acero de construcción embebida en su interior. Con el objetivo de evaluar el comportamiento frente a la corrosión se estudiaron aspectos termodinámicos y cinéticos. Los resultados mostraron que cementos comerciales mezclados con ceniza volante y escoria de alto horno, como los usados en esta investigación, presentan menor resistencia mecánica y a la corrosión que un cemento comercial.
\end{abstract}

PALABRAS CLAVE: Cemento comercial; Cemento hidráulico adicionado; Corrosión; Materiales cementantes suplementarios

Citation / Cómo citar este artículo: Gutiérrez-Junco, O.J., Pineda-Triana, Y., Vera-López, E. (2015) "Efecto de la incorporación de ceniza volante y escoria de horno alto en el comportamiento electroquímico de concretos de cemento comercial”. Rev. Metal. 51(4): e058. doi: http://dx.doi.org/10.3989/revmetalm.058.

\begin{abstract}
Effect of incorporation of fly ash and granulated blast furnace in the electrochemical behavior of concretes of commercial cement. This paper presents the findings of the research properties evaluation pastes of commercial cement (CPC), mixed with fly ash (FA) and granulated blast furnace slag (GBFS). Initially, the sample of 30 combinations were evaluated in terms of compressive strength to establish the optimal proportions from raw material. After that, four optimized blends were characterized during the setting and hardening process. Electrochemical tests were performed on concrete cylinders samples prepared with cementitious materials and a structural steel rod placed in the center of the specimen. With the objective to evaluate the performance before corrosion, thermodynamic and kinetic aspects were taken into consideration. The findings showed that commercial cements blended with fly ash and blast furnace slag as the ones used in this research presents a decreased behavior in mechanical and corrosion strength regarding to CPC.
\end{abstract}

KEYWORDS: Blended hydraulic cement; Commercial cement; Corrosion; Supplementary cementitious materials

Copyright: (C) 2015 CSIC. This is an open-access article distributed under the terms of the Creative Commons Attribution-Non Commercial (by-nc) Spain 3.0 License. 


\section{INTRODUCCIÓN}

La combinación de materiales cementantes suplementarios (SCMs) con cemento portland ordinario (OPC) ha mostrado importantes ventajas técnicas, económicas y ambientales (Jalal et al., 2015; Fernández-Carrasco et al., 2012). Sin embargo, la reactividad de la mezcla está directamente influenciada por los tipos, calidades y proporciones de las materias primas utilizadas (McCarter et al., 2013).

La ceniza volante (FA) es un subproducto de las estaciones termoeléctricas y la escoria granulada de horno alto (GBFS) un subproducto de la industria del acero. Ambos materiales son regulados y utilizados hoy en día para la fabricación de cementos adicionados (ASTM C989, 1999; ASTM C595, 2000; ASTM C311, 2000). No obstante, y de acuerdo con Lothenbach et al. (2011), el conocimiento de las relaciones entre la composición de estos materiales, sus productos de hidratación, y su impacto en el desarrollo de propiedades mecánicas y de durabilidad sigue siendo insuficiente.

Respecto a la escoria granulada, McCarter et al. (2013) y Fonseca et al. (2015), indican que el nivel de remplazo más utilizado está en el intervalo de $35-65 \%$ por peso del total de material cementante. El comité 233 del ACI (ACI 233R-95, 2000) explica el mecanismo de reacción del cemento adicionado con escoria, mencionando que el principal producto de hidratación es esencialmente el mismo producto principal formado cuando las pastas de OPC se hidratan, o sea silicato de calcio hidratado (CSH). Se produce entonces una densificación de la pasta de cemento, debido al relleno de poros capilares con gel CSH de baja densidad, que además de generar un concreto más impermeable, puede contribuir a la reducción de los procesos de corrosión debido al incremento de la resistividad del concreto (Song y Saraswathy, 2006).

Sobre la ceniza volante, Özbay et al. (2013), menciona que es el mineral de mezcla más comúnmente disponible entre los diferentes SCMs. Shaikh y Supit (2015), afirman que en aplicaciones generales el remplazo de OPC por FA está limitado a un intervalo de 15 a $20 \%$. De acuerdo con el informe técnico ACI 232.2R (ACI 232.2R-03, 2003), el proceso de reacción es similar a la hidratación del cemento portland ya que se produce CSH y aluminatos de calcio hidratados pero a partir de la reacción puzolánica entre la ceniza, el hidróxido de calcio y los álcalis en el concreto.

Flatt et al. (2012), exponen que la cinética de la hidratación de los cementos adicionados, no está dada por la superposición de las cinéticas de hidratación de sus componentes tomados individualmente. La mezcla de OPC con adiciones conduce a productos de hidratación más complejos que los del solo OPC, debido a la coexistencia de múltiples interdependientes y reacciones simultáneas (Fonseca et al., 2015).

Muchas investigaciones llevadas a cabo sobre el uso de la escoria y ceniza (solo escoria o combinación de escoria y ceniza) como sustitución parcial del OPC, indican la baja resistencia a la compresión a edades tempranas (1 a 7 días) debido a la lenta velocidad de hidratación de la escoria y ceniza. Sin embargo a edades tardías (después de los 28 días y hasta los 365 días) muestran mejores resultados que los concretos de solo OPC (Makhloufi et al., 2015; Jeong et al., 2015). De igual manera, se ha comprobado en otras investigaciones que las resistencias disminuyen gradualmente a medida que el nivel de reemplazo aumenta. Esto puede ser atribuido al hecho de que el OPC es substituido por adiciones minerales, lo cual conduce a la reducción del contenido de $\mathrm{CaO}$ disponible, y en consecuencia la formación inicial de gel CSH se inhibe (Bostanci et al., 2015). Similares conclusiones son mencionadas por Ahmaruzzaman (2010) y Juenger y Siddique (2015), sobre la sustitución parcial de OPC por ceniza. Ahmaruzzaman (2010) afirma que en general las propiedades mecánicas a largo plazo de los concretos con ceniza volante son más altas que las de los concretos con cemento portland debido a la reacción puzolánica; sin embargo, las tasas de fraguado y endurecimiento de los concretos con ceniza a edades tempranas son más lentas, debido a la dilución del cemento.

Algunas críticas a los cementos adicionados con materiales alternativos, mencionadas por Flatt et al. (2012), son sus bajas resistencias iniciales, las limitadas reservas de adiciones activas y los remplazos reducidos de adiciones no activas, o pobremente reactivas, sin que afecten de manera importante a la resistencia final. De igual manera, se cuestiona su resistencia a la carbonatación, fenómeno que compromete la protección a la corrosión del refuerzo del concreto, al propiciar mecanismos electroquímicos en el acero con intervención de solución acuosa (Bermúdez-Odriozola, 2007).

Por lo general, el concreto le da excelente protección a la corrosión al acero, debido a la alta alcalinidad que posee la pasta de cemento $(\mathrm{pH}>12,5)$, lo que hace que el refuerzo permanezca en un estado pasivo. El comité 222 del ACI (ACI 222.R-01, 2010) explica que esto ocurre al crearse una película de óxido de hierro adherida firmemente al acero, que lo protege de la corrosión. Pero esta protección puede fallar, debido por ejemplo a la acción del $\mathrm{CO}_{2}$, reduciendo la alcalinidad debido a la carbonatación (Song y Saraswathy, 2007). Esta reacción depende fundamentalmente de la humedad relativa ambiente, la temperatura, la permeabilidad del concreto y la concentración de $\mathrm{CO}_{2}$ (ACI 201.2R-01, 2000; Pacheco et al., 2012).

La carbonatación del concreto ha venido siendo investigada desde la mitad del siglo pasado hasta nuestros días, sin que se pueda afirmar que actualmente sea un proceso completamente entendido (Galán, 2011). La literatura sobre concretos de cemento adicionado con escoria y ceniza pone de manifiesto la reducción en la resistencia a la carbonatación, respecto a 
concretos de OPC, incrementándose el efecto con el aumento del nivel del reemplazo (Bostanci et al., 2015; Dong et al., 2015). La razón expuesta es la reducción de portlandita disponible en la estructura de poro debido a su consumo por la reacción puzolánica en el sistema cementante.

Según Schneider et al. (2011), el desarrollo de cementos con varios constituyentes principales, sustituyendo parcialmente el contenido de clinker con SCMs, ha hecho que la producción de OPC disminuya en el tiempo. Un caso particular de estudio, es el caso Colombiano, en el cual las cementeras denominaban su producto acorde a la norma de cemento portland ASTM C150 (1999), pero elaboraban el cemento, en algunos casos, usando un modelo de cemento adicionado. Con la expedición de la norma técnica Colombiana NTC 121 de 2014 (NTC 121, 2014), se rectificó la incongruencia mencionada. En ese contexto, el presente trabajo pretende evaluar un conjunto de propiedades de un cemento diseñado sin restricción en su composición, comercializado en Colombia como portland Tipo I (CPC), y compararlo con las prestaciones de cementantes elaborados a partir de la combinación del mismo $\mathrm{CPC}$ con adiciones de ceniza y escoria.

\section{MATERIALES Y MÉTODOS}

\subsection{Materiales}

Como materias primas, se utilizó cemento comercial (CPC), fabricado en Colombia como portland tipo I (ASTM C150, 1999) y tipo GU (ASTM C1157/ C1157M, 2011), producido por una cementera nacional: ceniza volante (FA) obtenida de la central termoeléctrica Termopaipa (Boyacá, Colombia) y escoria granulada de alto horno (GBFS) obtenida en la empresa Acerías Paz del Rio S.A. (Boyacá, Colombia). Antes de ser usada, la escoria fue deshidratada y molida en seco, luego tamizada a través del tamiz de $250 \mu \mathrm{m}$. Además, se utilizaron agregados calizos (TMN 25,4 mm), arena lavada (Módulo de Finura 2.24) y arena normalizada 20-30 (Equivalente arena de Ottawa). Se utilizó agua potable, cuyos iones y características fisicoquímicas están dentro de los rangos permitidos por la norma técnica colombiana NTC 3459 (NTC 3459, 2001) ( $\mathrm{pH}=7,2$, Cloruros $=4,2 \mathrm{mg} \mathrm{Cl} / \mathrm{L}$, Sulfatos=4,6 $\mathrm{mg} \mathrm{SO}_{4} / \mathrm{L}$, Alcalinidad=7,7 $\mathrm{mg} \mathrm{CaCO}_{3} / \mathrm{L}$ ). Finalmente, se utilizó aditivo plastificante. El acero utilizado fue uno de baja aleación, ASTM A706, grado 60 y diámetro $13 \mathrm{~mm}$ para refuerzo de concreto (Composición: $\mathrm{C}=0,30 \%$, $\mathrm{Mn}=1,19 \%, \mathrm{P}=0,014 \%, \mathrm{~S}=0,036 \%$ y $\mathrm{Si}=0,22 \%$ ).

\subsection{Preparación de probetas de ensayo}

Se elaboraron 270 probetas de pasta cementante de dimensiones $50,8 \mathrm{~mm} \times 50,8 \mathrm{~mm}$, repartidas en 30 combinaciones de CPC, FA y GBFS y tres fechas de prueba. Se usó una relación agua/material cementante $\left(\mathrm{H}_{2} \mathrm{O} / \mathrm{mc}\right)$ fija de 0,277 , aumentando la fluidez de las mezclas secas (mezclas con FA) con el aditivo. La relación $\mathrm{H}_{2} \mathrm{O} / \mathrm{mc}$ fija se determinó a partir del ensayo de consistencia normal del CPC siguiendo las pautas de la norma ASTM C187:98 (ASTM C187-98, 1998).

Se elaboraron 48 probetas de ensayos de mortero, de dimensiones $50,8 \mathrm{~mm}$ x 50,8 $\mathrm{mm}$, repartidas en 4 combinaciones de CPC, FA y GBFS y cuatro fechas de prueba. Se usó la arena normalizada y una relación $\mathrm{H}_{2} \mathrm{O} / \mathrm{mc}$ fija de 0,56 correspondiente a la fluidez de $110 \pm 5 \%$ para el CPC (ASTM C230/ C230M, 1998).

Las demás condiciones de elaboración de las probetas de ensayo se definieron a partir de la norma ASTM C109M:99 (ASTM C109/C109M, 1999).

Adicionalmente se elaboraron 60 probetas de ensayo de concreto, de geometría cilíndrica, de $100 \mathrm{~mm}$ de diámetro por $200 \mathrm{~mm}$ de altura, repartidos en cuatro combinaciones de CPC, FA y GBFS y cinco fechas de prueba. La relación entre cementante, arena lavada y grava fue de 1:2.4:3.5 y la relación $\mathrm{H}_{2} \mathrm{O} / \mathrm{mc}$ de 0,58 . La norma de referencia fue la ASTM C192/C192M-00 (ASTM C192/C192M, 2000).

Un total de tres probetas fueron moldeadas por cada tipo de mezcla, y por cada fecha de ensayo. Los cubos se colocaron en un estado similar de saturación de agua a las 24 horas y se mantuvieron de esa manera hasta la fecha del ensayo. Por su parte los cilindros fueron curados con humedad durante 28 días, luego fueron localizados en un ambiente de exposición atmosférica bajo techo y ventilado, en la ciudad de Tunja-Colombia, hasta el día de la falla. Complementariamente 16 cilindros de concreto, reforzados, se elaboraron para las pruebas electroquímicas.

\subsection{Técnicas de caracterización y procedimiento experimental}

\subsubsection{Técnicas de caracterización de materias primas}

Se utilizó microscopía electrónica de barrido (SEM), con el equipo ZEIIS Serie EVO | MA10 y análisis de fisisorción de nitrógeno (BET) a $-196{ }^{\circ} \mathrm{C}$, con previa limpieza de la muestra a $100{ }^{\circ} \mathrm{C}$ con el equipo Micromeritics ASAP 2020. Se realizaron mediciones de superficie específica con el aparato de Blaine (ASTM C204, 1996) y densidad (ASTM C188, 1995). Asimismo se utilizó espectrometría de fluorescencia (XRF), en un equipo PANalytical MiniPal 2, con tubo de Rhodio, trabajando a $10 \mathrm{kV}$ y 0,002 mA, durante 180 segundos. Adicionalmente a la ceniza volante se le practicaron los llamados análisis inmediatos del carbón y del coque (ASTM D3173, 2011), los cuales corresponden a la evaluación de los porcentajes de humedad, ceniza, materia volátil y carbono fijo. 


\subsubsection{Procedimiento experimental}

Como punto de partida para la dosificación de las probetas de mortero y concreto, se realizó una serie de pruebas de resistencia mecánica en cubos de pasta cementante. El objetivo fue encontrar la dosificación óptima de FA y GBFS en combinaciones tanto binarias como ternarias con CPC. En total se evaluó la resistencia a la compresión de 30 dosificaciones de pasta con diferente contenido de CPC, FA y GBFS, a 3, 28 y 56 días de elaboración. La comparación entre las resistencias de las mezclas permitió definir cuatro composiciones para ser usadas en la elaboración de los morteros y concretos.

Se realizaron ensayos de caracterización a los cuatro tipos de pasta seleccionados: determinación de la consistencia normal con el aparato de Vicat, siguiendo el procedimiento de la norma ASTM C187 (ASTM C187-98, 1998), el cual evalúa la cantidad de agua requerida para obtener una pasta de cementante con una consistencia estandarizada, considerada óptima en términos de fluidez y plasticidad; tiempos de fraguado con la norma ASTM C191 (ASTM C191, 1999), para caracterizar el tiempo en que la pasta tarda en pasar de estado plástico a sólido; y medición del calor de hidratación, a partir del comportamiento térmico de las pastas, de acuerdo con las recomendaciones del método semi-adiabático de la norma BS EN 196-9 (BS EN 196-9, 2003).

Adicionalmente, el programa de pruebas incluyó el control de la resistencia mecánica de los morteros (ASTM C109/C109M, 1999) a 3, 7, 28 y 56 días y de los concretos (ASTM C39/C39M, 2001), a 3, 7, 28, 56 y 118 días de elaboración, usando las cuatro composiciones cementantes elegidas, con miras a evaluar la participación de los SCMs.

Finalmente se realizaron pruebas electroquímicas durante el proceso de endurecimiento de los cilindros de concreto elaborados con los cementantes y con una varilla de acero de construcción ASTM A706 embebida en su interior. Con el objetivo de evaluar el comportamiento frente a la corrosión se estudiaron aspectos termodinámicos y cinéticos, usando curvas de polarización potenciodinámicas corriente-voltaje y aplicando el método de resistencia a la polarización (LPR). Las mediciones se realizaron a 3, 28, 42, 56, 70, 84, 98 y 118 días de edad de las probetas. Los cilindros estuvieron sumergidos en agua hasta el día 28, y luego fueron puestos en condición ambiental de la ciudad de Tunja, con atmósfera aireada y bajo techo (condición denominada b1). También se realizó el estudio con deterioro por carbonatación, en cilindros elaborados con las características mencionadas, pero sometidos a carbonatación acelerada en una atmósfera artificial (humedad relativa de $60 \pm 10 \%$, concentración de $\mathrm{CO}_{2}$ de $1 \%$, y temperatura $21 \pm 2{ }^{\circ} \mathrm{C}$ ) a partir del día 42 (condición denominada b2).
La norma ASTM G102 (ASTM G102-89, 1999), expone los cálculos para las mediciones electroquímicas. Éstos comprenden la evaluación de la constante de Stern-Geary (B) y el uso de la resistencia a la polarización $\left(R_{P}\right)$ para establecer la densidad de corriente de corrosión $\left(\mathrm{i}_{\text {corr }}\right)$, la corriente anódica total $\left(\mathrm{I}_{\text {corr }}\right)$ y la velocidad de corrosión $(\mathrm{CR})$.

El proceso de evaluación electroquímica se describe en el apartado 3.3, donde se relaciona las velocidades de corrosión encontradas con el estado de pasividad/actividad de las varillas.

\section{RESULTADOS Y DISCUSIÓN}

\subsection{Caracterización de materias primas}

En la Fig. 1 se presentan micrografías (SEM) de las materias primas cementantes. El CPC muestra variedad de tamaños de partículas dentro de los rangos usuales. La FA muestra sus típicas partículas esféricas con superficies suaves. Se destaca el mayor tamaño estructural de la GBFS respecto a los otros dos cementantes junto con su morfología angular.

En cuanto a las superficies específicas evaluadas con el permeabilímetro de Blaine, los resultados encontrados fueron para el CPC, $4230 \mathrm{~cm}^{2} \mathrm{~g}^{-1}$, para la FA, $4640 \mathrm{~cm}^{2} \mathrm{~g}^{-1}$, y para la GBFS, $1240 \mathrm{~cm}^{2} \mathrm{~g}^{-1}$. La GBFS, por su menor área superficial, presenta la condición más desfavorable para la reacción hidráulica.

En el análisis de fisisorción de nitrógeno, siguiendo el tratamiento matemático propuesto por BET (Brunauer, Emmett y Teller), se obtuvieron los resultados indicados en la Tabla 1.

La reactividad de los materiales cementantes depende de una combinación de factores, tales como la composición química, mineralógica y la finura. Sobre este último, el método de finura Blaine y el análisis de fisisorción coinciden en que la GBFS tiene la menor área superficial. Por su parte la FA, con su mayor área superficial y mayor volumen de poro, hace que las mezclas que contienen CPC-FA demanden más agua para generar manejabilidades comparables a las de las mezclas de solo CPC y de CPC-GBFS. Esta es la razón por la cual fue necesario usar el aditivo plastificante en las pastas con FA, logrando así las manejabilidades requeridas sin agua adicional.

La evaluación de las densidades reportó para el CPC 2,90 $\mathrm{g} \mathrm{cm}^{-3}$, para la FA 2,06 $\mathrm{g} \mathrm{cm}^{-3}$, y para la GBFS 2,86 $\mathrm{g} \mathrm{cm}^{-3}$. El valor inferior de la densidad del CPC comparado con el $\sim 3,15 \mathrm{~g} \mathrm{~cm}^{-3}$ del OPC (portland puro), da un indicativo del alto contenido de adiciones original del CPC.

En la Tabla 2 se presenta el análisis de óxidos de los materiales cementantes, evaluados mediante espectrometría de fluorescencia de rayos X (XRF).

El CPC muestra altos contenidos de $\mathrm{CaO}$ y $\mathrm{SiO}_{2}$, compuestos importantes para la generación de silicatos de calcio hidratados. En la FA se destaca el 
(a)

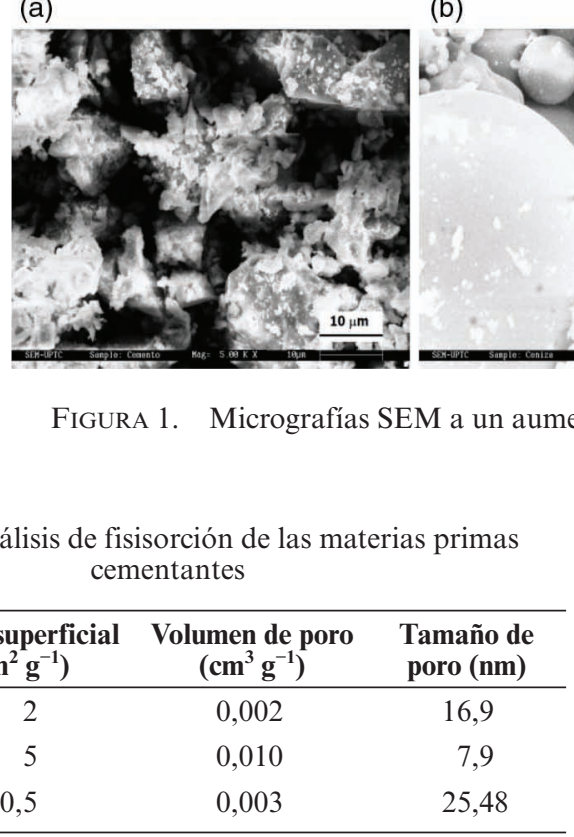

TABla 2. Composición química de los materiales cementantes ( $\%$ en peso)

\begin{tabular}{lccr}
\hline & CPC & FA & GBFS \\
\hline $\mathrm{SiO}_{2}$ & 16,51 & 60,78 & 33,72 \\
$\mathrm{CaO}$ & 60,5 & 0,73 & 43,93 \\
$\mathrm{Al}_{2} \mathrm{O}_{3}$ & 3,6 & 22,3 & 16,26 \\
$\mathrm{MgO}$ & 0,99 & 0,54 & 1,36 \\
$\mathrm{Fe}_{2} \mathrm{O}_{3}$ & 1,73 & 4,8 & 1,07 \\
$\mathrm{SO}_{3}$ & 2,7 & $-\mathrm{a}$ & 0,99 \\
$\mathrm{MnO}$ & 0,03 & $-\mathrm{a}$ & 1,36 \\
$\mathrm{~K}_{2} \mathrm{O}$ & 0,28 & 1,57 & 0,32 \\
$\mathrm{TiO}_{2}$ & 0,13 & 1,09 & 0,49 \\
\hline
\end{tabular}

a $-=$ no determinado.

TABla 3. Características físico-químicas de la ceniza volante

\begin{tabular}{lc}
\hline Parámetro & \% en peso \\
\hline${\text { Humedad }(\mathrm{H})^{\mathrm{a}}}^{\mathrm{b}}$ & 0,80 \\
Ceniza residual $^{\mathrm{b}}$ & 89,04 \\
${\text { Materia volátil }(\mathrm{MV})^{\mathrm{c}}}^{\mathrm{c}}$ & 1,70 \\
${\text { Carbono fijo }(\mathrm{CF})^{\mathrm{d}}}^{\mathrm{e}}$ & 8,46 \\
Total inquemados $^{\mathrm{e}}$ & 10,16 \\
Total de pérdidas por calcinación $^{\mathrm{f}}$ & 10,96
\end{tabular}

${ }^{\mathrm{a} A S T M}$ D3173; ${ }^{\mathrm{b}}$ ASTM D3174; ${ }^{\mathrm{c} A S T M}$ D3175; ${ }^{\mathrm{d} A S T M}$

$\mathrm{D} 3172 ;{ }^{\mathrm{e}} \mathrm{CF}+\mathrm{MV} ;{ }^{\mathrm{f}} \mathrm{CF}+\mathrm{MV}+\mathrm{H}$.

$\mathrm{SiO}_{2}$ y el $\mathrm{Al}_{2} \mathrm{O}_{3}$. Atendiendo al contenido de $\mathrm{CaO}$, la FA se puede clasificar como baja en $\mathrm{CaO}$ (menor a $10 \%$ ), siendo esto característico en la combustión de antracita y carbones bituminosos. La actividad hidráulica de las cenizas es mayor conforme aumenta el contenido de $\mathrm{CaO}$. La GBFS se puede clasificar como una escoria básica al tener una relación $\mathrm{CaO}$ / $\mathrm{SiO}_{2}$ superior a 1 , lo cual es favorable en términos de reactividad (potencial hidráulico).

Los resultados de los análisis inmediatos aplicados a la FA se presentan en la Tabla 3.

Considerando los requisitos químicos de la norma ASTM C618 (2005), la FA se puede clasificar como de tipo F. Esta norma presenta un límite máximo de $6 \%$ para la pérdida al fuego, no obstante, establece también la posibilidad de usar puzolanas hasta con un $12 \%$ de pérdida al fuego si se suministran registros de un comportamiento aceptable, o los resultados de pruebas de laboratorio. El contenido de carbón en la FA es un resultado de la combustión incompleta del carbón y aditivos orgánicos usados en el proceso de recolección. En su gran mayoría el total de inquemados es carbón sin quemar.

\subsection{Elección y caracterización de mezclas optimizadas}

A partir de los resultados de resistencia a la compresión de los cubos de pasta a 3,28 y 56 días, se eligieron cuatro composiciones de cementante para ser utilizadas en la elaboración de los morteros y concretos (Tabla 4). En general, la resistencia a la compresión aumentó con el contenido de CPC y el porcentaje de GBFS resultó más favorable que el de FA.

La Tabla 5, muestra los resultados de los ensayos de consistencia normal y tiempos de fraguado. El resultado de consistencia normal de la pasta con cementante a3 muestra el efecto de la alta superficie específica de la FA $\left(4640 \mathrm{~cm}^{2} \mathrm{~g}^{-1}\right)$, demandando la mayor cantidad de agua. Por su parte la GBFS con menor superficie específica $\left(1240 \mathrm{~cm}^{2} \mathrm{~g}^{-1}\right)$, disminuyó la cantidad de agua requerida para obtener la consistencia normal en la pasta de cementante a2. El resultado de la pasta de cementante a4 sumó los efectos de la FA y la GBFS equiparándose con la pasta de referencia a1. Respecto a los tiempos de fraguado, la mezcla a4 (la más adicionada), presentó 
TABla 4. Cementantes seleccionados para pruebas en morteros y concretos

\begin{tabular}{lc}
\hline Nombre del Cementante & \multicolumn{1}{c}{ Materiales } \\
\hline a1 & $100 \%$ CPC (Referencia) \\
a2 & $80 \%$ CPC $+20 \%$ GBFS \\
a3 & $90 \% \mathrm{CPC}+10 \%$ FA \\
a4 & $70 \% \mathrm{CPC}+20 \%$ GBFS $+10 \%$ FA \\
\hline
\end{tabular}

TABLA 5. Ensayos realizados en pasta (consistencia normal y tiempos de fraguado)

\begin{tabular}{lccc}
\hline Cementante & $\begin{array}{c}\text { Consistencia } \\
\text { normal (\%) }\end{array}$ & $\begin{array}{c}\text { Tiempo fraguado } \\
\text { inicial (h) }\end{array}$ & $\begin{array}{c}\text { Tiempo fraguado } \\
\text { final (h) }\end{array}$ \\
\hline a1 & 28,6 & 4,2 & 9,8 \\
a2 & 26,0 & 4,2 & 9,8 \\
a3 & 30,0 & 4,1 & 9,7 \\
a4 & 28,5 & 4,7 & 10,7 \\
\hline
\end{tabular}

la menor velocidad e intensidad de las reacciones de hidratación, retrasándose tanto el inicio como el final del fraguado. La velocidad de endurecimiento fue similar para las otras mezclas.

$\mathrm{El}$ registro de cambio de temperatura en las pastas para la evaluación del calor de hidratación se presenta en la Fig. 2. Se observa que los tiempos de fraguado inicial y final concuerdan con el inicio del aumento de la pendiente de cambio de temperatura y con el valor pico de temperatura respectivamente. Como resultado se tiene que el calor desprendido, en la pasta de solo CPC, es más alto con respecto a las de CPC mezclado con adición. La pasta de cementante al presenta la mayor energía interna, lo que hace que sea el material de mayor reactividad y por tanto el más inestable y con mayor retracción. Le sigue la pasta de cementante a3 y luego la de a2. La pasta de cementante a4 tiene el menor calor de hidratación, lo que conlleva un menor riesgo de fisuramiento por contracción, cualidad deseada para estructuras de concreto en masa.
Respecto a la evaluación en morteros, éstos se realizaron con la arena normalizada y con la cantidad de agua evaluada mediante el ensayo de fluidez del mortero de referencia (cementante a1). Hay que destacar la mayor relación $\mathrm{a} / \mathrm{mc}$ requerida para lograr fluidez de $110 \pm 5 \%$ del mortero de referencia respecto a un mortero de cemento portland puro $(0,56$ vs $\sim 0,485)$, indicativo de las adiciones originarias en el CPC. Paralelamente también se hicieron ensayos en concretos.

La Fig. 3 muestra la resistencia a la compresión encontrada en los morteros y concretos con cementantes a1, a2, a3 y a4. En general, las probetas con cementante a2, a3 y a4 presentaron, de manera concordante, disminución en sus propiedades mecánicas, con respecto a las probetas de referencia que utilizan solo el cemento de producción nacional (cementante a1). Esto puede justificarse en la inferior reactividad del CPC en combinación con las adiciones, indicando que el porcentaje de adición total en las mezclas (correspondiente a la que originariamente trae el CPC y la adicionada), es demasiado alto respecto a la cantidad y tipo de clinker presente en el CPC. Las mezclas con cementante a4 presentaron el menor potencial hidráulico debido al mayor contenido de adición.

El efecto del tipo de cementante en el comportamiento mecánico del mortero y del concreto, concordó con los resultados encontrados en las pastas. Con menor concentración de adición las mezclas con cementante a3 lograron superar las resistencias de las mezclas con a2, a pesar del alto contenido de inquemados de la FA.

A edades tempranas, durante los primeros 28 días, las mezclas con solo CPC (en pasta, mortero y concreto) presentaron el mayor aumento de resistencia, indicando que la reacción hidráulica y puzolánica de las mezclas con adiciones es más lenta y menos efectiva, mientras que los resultados a mediano y largo plazo, de todas las mezclas, mostraron una tendencia similar.

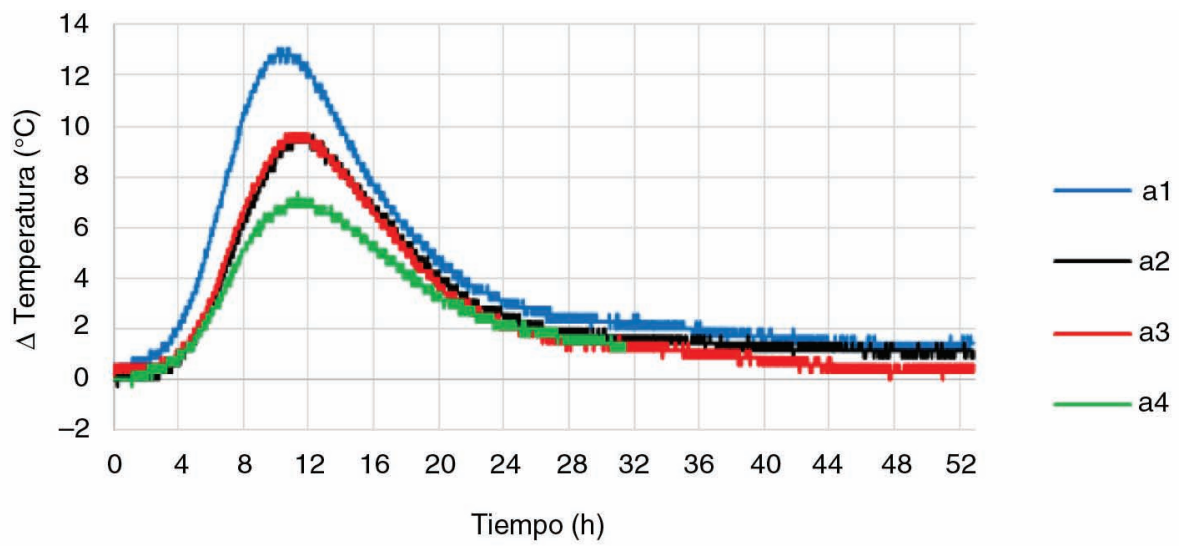

FIgURA 2. Variación de la temperatura en las pastas en función del tiempo. 
(a)

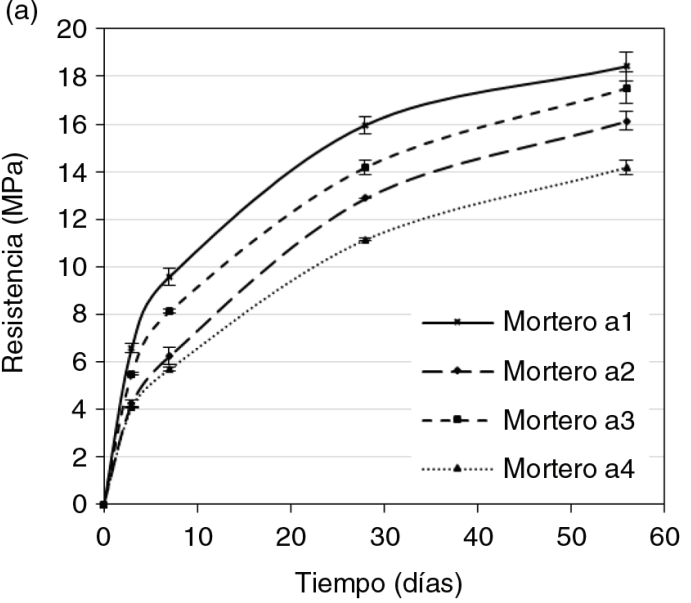

(b)

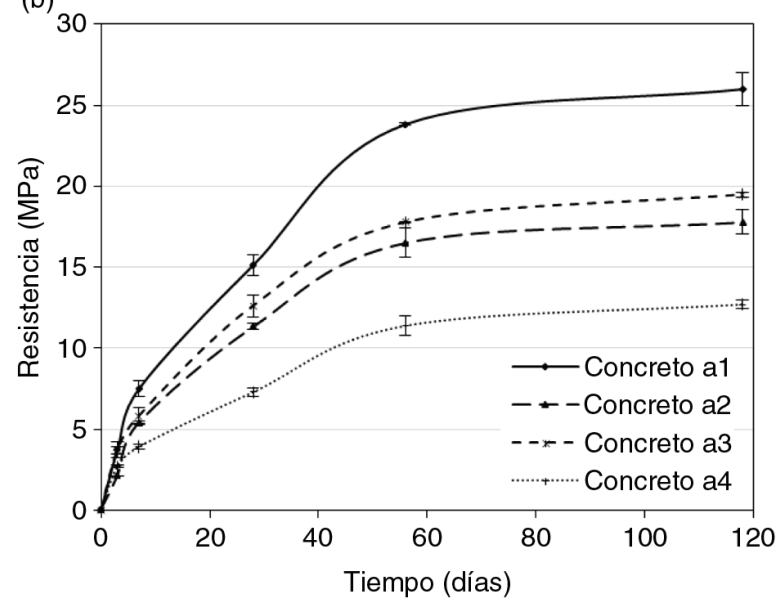

Figura 3. Evolución de resistencia de: (a) morteros y (b) concretos.

\subsection{Evaluación electroquímica}

La Fig. 4 describe el montaje experimental utilizado, el cual consistió en una configuración de celda clásica de tres electrodos sumergida parcialmente en agua potable. El electrodo de trabajo corresponde a una varilla de acero ASTM A706, grado 60 y diámetro $13 \mathrm{~mm}$, maquinada y pulida (para considerar una superficie estándar), y protegida con anticorrosivo epóxico, excepto en el área expuesta a la corrosión. El área expuesta del electrodo de trabajo fue de $40,8 \mathrm{~cm}^{2}$ y fue localizada a la mitad de la altura de los cilindros. El electrodo de referencia (pseudoelectrodo de referencia) consistió en una varilla de acero inoxidable grado AISI 304 diámetro $6,4 \mathrm{~mm}$, ajustado a las normas ASTM A276 (2010) y ASTM A262 (2002). Una lámina de acero inoxidable, de las mismas características del acero del electrodo de referencia, hizo las veces de contraelectrodo.

La evaluación de la corrosión se realizó elaborando curvas de polarización potenciodinámicas corriente-voltaje y aplicando el método de resistencia lineal a la polarización (LPR). Las curvas de polarización potenciodinámicas se obtuvieron a una velocidad de barrido de $5 \mathrm{mV} \mathrm{s}^{-1}$ en un rango de polarización total de $400 \mathrm{mV}$ que se distribuye alrededor del potencial de corrosión. En las pruebas LPR el potencial se varió entre $\pm 30 \mathrm{mV}$ respecto al Ecorr y la velocidad de barrido fue de $0,17 \mathrm{mV} \mathrm{s}^{-1}$ acorde con lo recomendado por la norma ASTM G59 (ASTM G59-97, 2003). Para la ejecución de las pruebas se usó un equipo Gamry referencia 750 y un software de control Gamry Framework Versión 5.67. En todos los casos se utilizaron dos probetas para promediar los resultados.

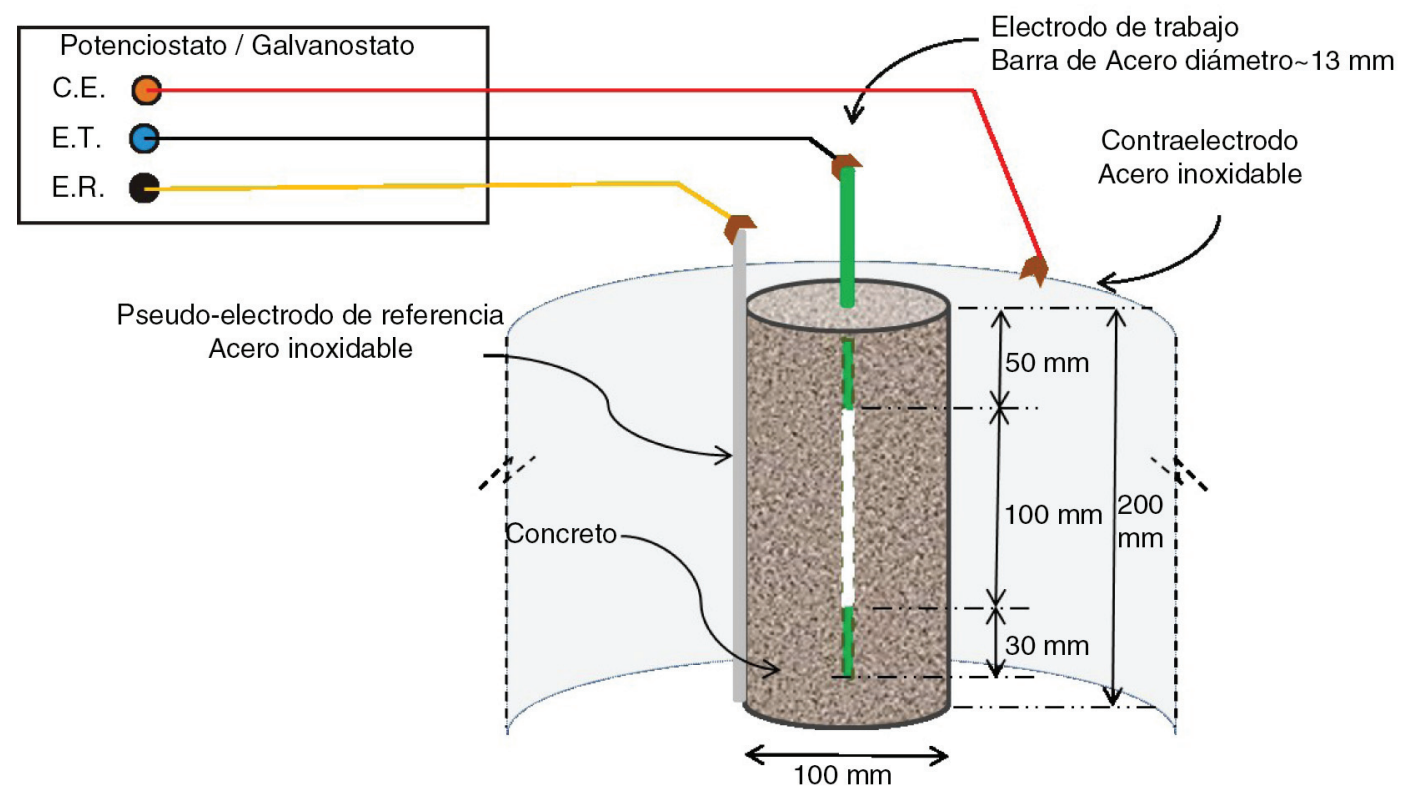

FIGURA 4. Geometría de la probeta y disposición experimental para pruebas electroquímicas. 


\subsubsection{Curvas de polarización potenciodinámicas corriente-voltaje}

Las curvas de polarización mostraron el estado de pasividad/actividad de los aceros embebidos en los cilindros de concreto durante la investigación. Grandes pendientes de la reacción anódica denotan estabilización de la corriente respecto al cambio de potencial, conllevando a estados donde el acero está protegido por una película pasiva. Bajas pendientes (o intermedias), por el contrario, implican ausencia de esta protección.

La Fig. 5 muestra la pasivación en el acero con cementante a1, y las bajas condiciones cinéticas (pendientes intermedias de las reacciones anódicas) en las probetas a2, a3, y a4, detectadas a 118 días en condición b1. En contraste la Fig. 6 muestra el comportamiento de los concretos en condición b2 (carbonatados artificialmente) a 118 días. Se evidencia el estado activo, susceptible a la corrosión, de las probetas con cementante a4, y en menor proporción de las probetas con cementante a2 y a3. Hay que destacar las bajas pendientes de las reacciones anódicas y el mayor desplazamiento hacia la derecha de la curva correspondiente a la mezcla a4, indicando una mayor corriente de corrosión $\left(\mathrm{I}_{\text {corr }}\right)$. En este gráfico el concreto a1 presenta el mayor potencial de corrosión $\left(\mathrm{E}_{\text {corr }}\right)$, la menor corriente anódica, la menor cinética, y por tanto ofrece la menor velocidad de corrosión respecto a los demás cementantes.

\subsubsection{Resistencia de polarización}

La estimación de las velocidades de corrosión con el método de resistencia de polarización se presenta en la Fig. 7, en la que se indica el promedio y el

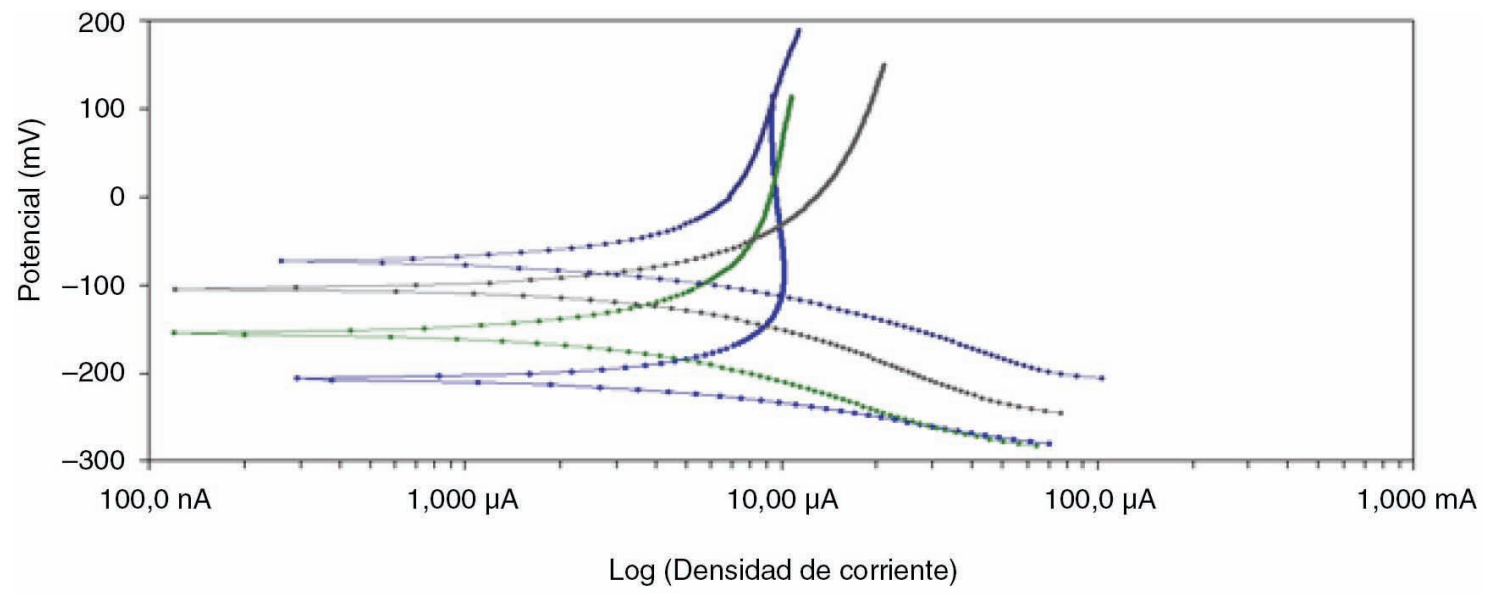

FIGURA 5. Curvas de polarización potenciodinámicas corriente-voltaje desarrolladas a 118 días en probetas en condición b1 con cementante: (a) a1; (b) a2; (c) a3 y (d) a4.

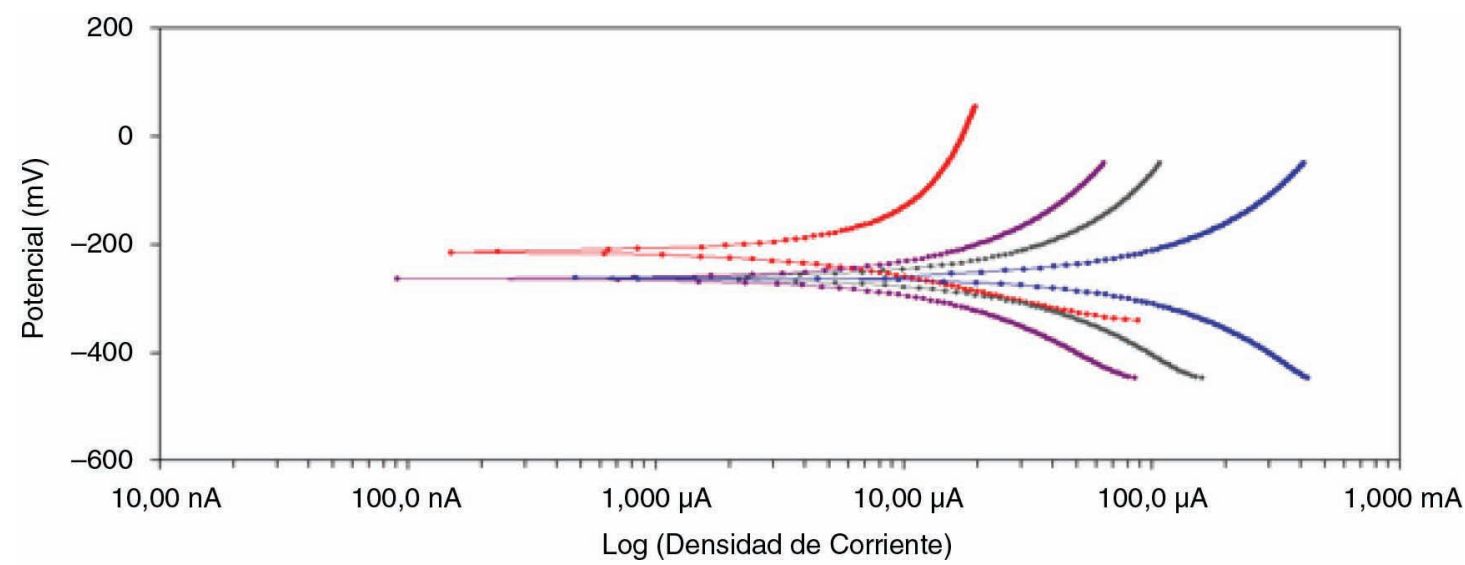

FiguRa 6. Curvas de polarización potenciodinámicas corriente-voltaje desarrolladas a 118 días en probetas en condición b2 con cementante: (a) a1; (b) a2; (c) a3 y (d) a4. 


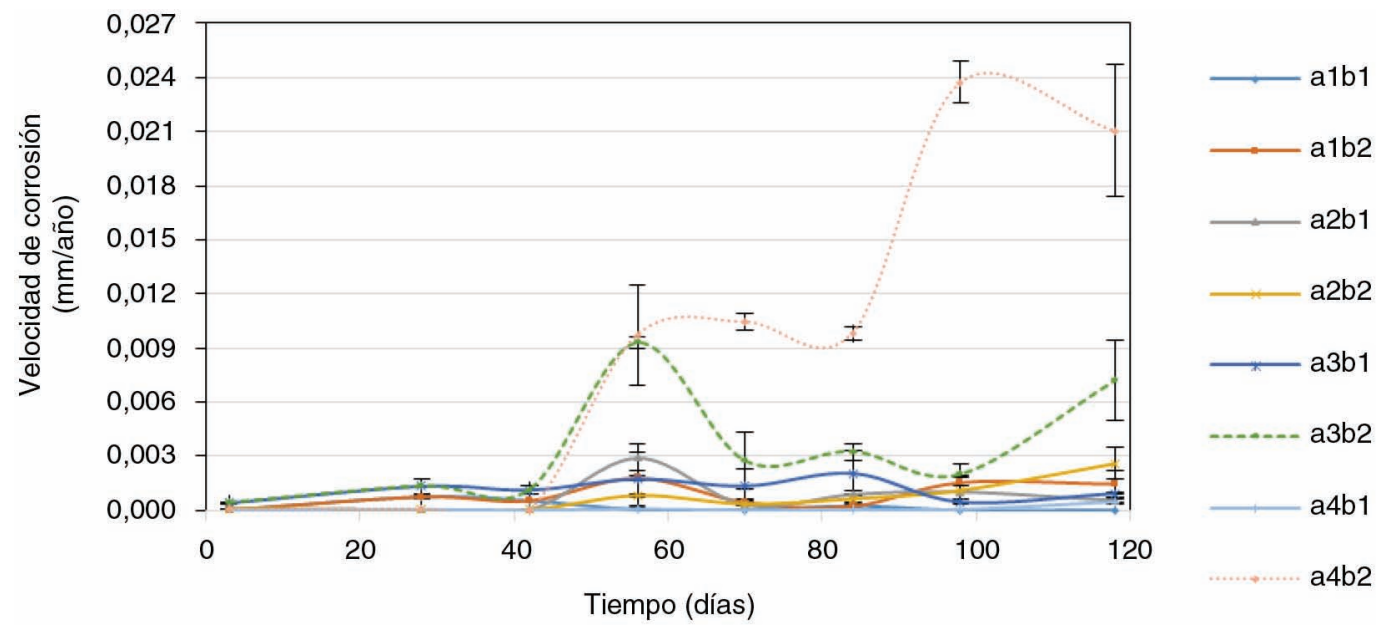

FIgURA 7. Comportamiento promedio de la velocidad de corrosión evaluada con la técnica LPR para las cuatro mezclas y las dos condiciones de almacenamiento en laboratorio.

rango de los valores encontrados. Con esta técnica se evidencia un importante aumento de la velocidad de corrosión en las probetas con cementante a4, debido a la carbonatación artificial (condición b2). La carbonatación acelerada hizo que disminuyera la resistencia a la polarización de todas las probetas a 118 días, comprometiendo especialmente la resistencia en los cilindros con cementante a4. Este resultado concuerda con lo hallado en las pruebas con curvas de polarización potenciodinámicas corriente-voltaje. En definitiva se detectó a 118 días bajas velocidades de corrosión en las probetas alb1, a2b1 a3b1, y a4b1, condiciones intermedias a bajas, en las probetas a1b2, a 2b2, y a3b2, y altas velocidades de corrosión en las probetas a4b2. De las probetas que tuvieron comportamiento intermedio a bajo, las a $3 \mathrm{~b} 2$ adquirieron las mayores velocidades de corrosión, y las a1b2 las menores.

\subsubsection{Análisis electroquímico e inspección visual}

Finalizado el estudio electroquímico se partieron los cilindros de concreto a la mitad, mediante tracción indirecta, para exponer las varillas y hacer un reconocimiento visual de los aceros (Fig. 8). Se encontró correspondencia entre los niveles de corrosión obtenidos con inspección directa y los registrados con electroquímica. La magnitud de la carbonatación de los cilindros se identificó mediante la coloración de la superficie interna del concreto con un indicador de $\mathrm{pH}$.

La inspección visual realizada a 118 días mostró como resultado principal la desventaja del cementante a4 para proteger las varillas en el ambiente de carbonatación artificial. En la condición ambiental b1 no se evidenció corrosión a simple vista en ninguno de los cuatro tipos de cementante estudiados (Fig. 8a). Para la condición b2 (con carbonatación acelerada hasta el día de la inspección), no se evidenció corrosión en las probetas con cementante al de 118 días. Por su parte las probetas con cementante a2 y a3 mostraron zonas de corrosión localizada de magnitud semejante (Fig. 8 b y c), mientras que las probetas con cementante a 4 presentaron corrosión generalizada en toda la zona de exposición de los aceros (Fig. 8d). Estos resultados concuerdan con el avance de la carbonatación en los concretos, detectado con el color violeta producido por el indicador ácido-base. Se complementa el anterior análisis, con las curvas de polarización potenciodinámicas y de velocidad de corrosión, donde hay evidencia que los concretos con cementante adicionado (a2, a3, y a4), en condición carbonatada, presentan un comportamiento menos favorable que el concreto con solo CPC (cementante a1).

Las pruebas de composición física, química y mineralógica de las materias primas permiten hacer las siguientes observaciones: se identificó contenido de calcita en el CPC (16\%); la pérdida por ignición para el CPC fue de $13,45 \%$ mayor a la permitida por la clasificación estándar ASTM C150 (1999); se encontraron superficies específicas Blaine en el CPC mayores a las típicas encontradas en un OPC $\left(\sim 3818 \mathrm{~cm}^{2} \mathrm{~g}^{-1}\right)$, y densidades del CPC menores a las del cemento portland ordinario; se encontró una menor proporción de contenido amorfo en la FA $(33 \%)$ respecto a la GBFS $(97 \%)$, y como es típico, mayor contenido de $\mathrm{CaO}$ en la GBFS que en la FA. Con estos resultados al parecer la GBFS tiene un mejor comportamiento debido a que es una adición más activa, y fundamentalmente se detecta la presencia de adiciones incorporadas de fábrica en el CPC, las cuales disminuyen la posibilidad de reactividad de la FA y la GBFS agregadas en este estudio. Adicionalmente el bajo contenido amorfo, el bajo contenido de $\mathrm{CaO}$, y los altos inquemados de la $\mathrm{FA}$, 

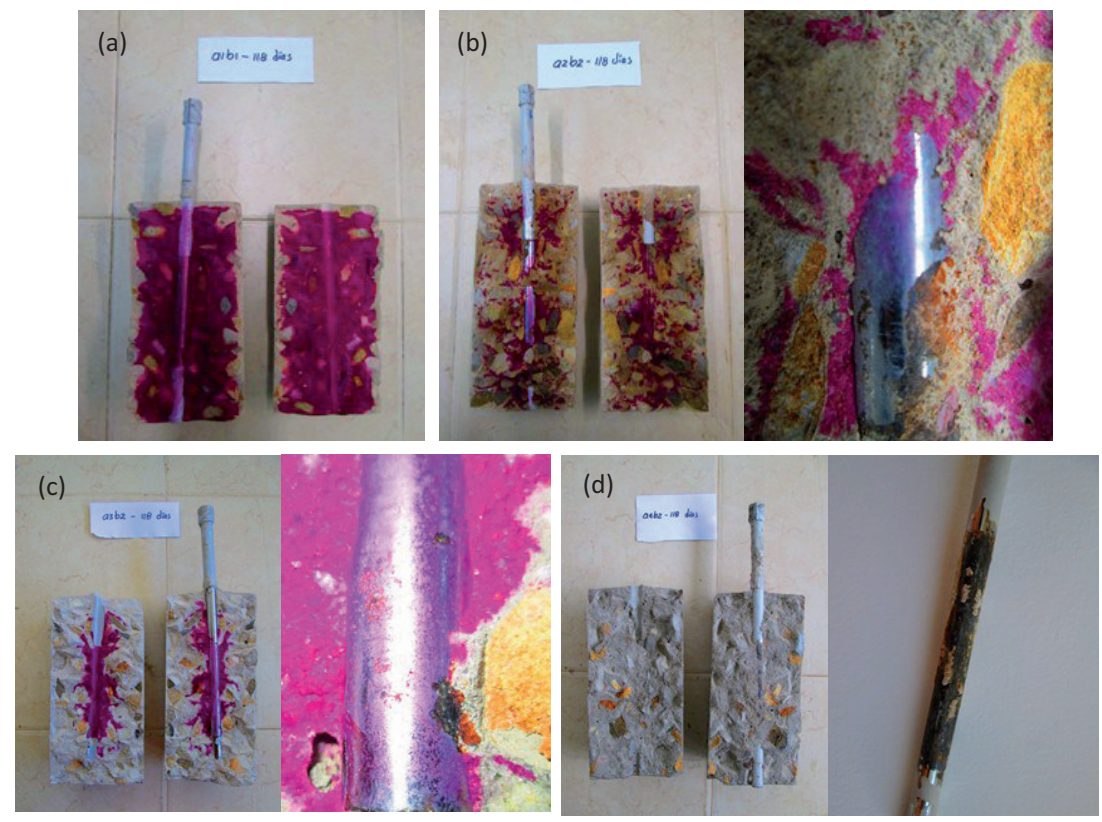

FIGURA 8. Inspección directa del estado de corrosión: (a) a1b1-118 días, sin corrosión a la vista; (b) a2b2-118 días, zonas de corrosión localizada; (c) a3b2-118 días, zonas de corrosión localizada y (d) a4b2-118 días, zona de corrosión generalizada.

comprometieron desfavorablemente su actividad puzolánica. Bajo estas circunstancias se puede interpretar que a mayor contenido de adición mayor cantidad de GBFS y FA no reactiva, menor cantidad de $\mathrm{CSH}$, y también menor cantidad de portlandita en las mezclas hidratadas. Esto explica el porqué, bajo carbonatación acelerada, el concreto con cementante al ofreció la mayor protección, los concretos con cementante a 2 y a 3 un comportamiento intermedio, y que el concreto de cementante a4 haya prestado la menor resistencia a la carbonatación, y por tanto a la corrosión. De la misma manera, la limitada generación de CSH afectó a la resistencia mecánica de pastas, morteros y concretos. El mecanismo de hidratación también se vio influenciado, aunque en menor proporción, por otros factores como el tamaño de las partículas y el área superficial específica de la FA y la GBFS molida.

\section{CONCLUSIONES}

- La FA y la GBFS, a pesar de ser consideradas adiciones activas, no adquirieron la capacidad suficiente de reacción con el agua en ambiente alcalino y con el hidróxido cálcico disponible de la hidratación del CPC, para obtener mayores resistencias a las presentadas por las mezclas de solo CPC. En todos los casos se mostró pérdida en la resistencia mecánica, evidenciando la formación de productos menos cohesivos y estables.

- Se evidenciaron altos contenidos de adiciones en el CPC, incorporados en fábrica, los cuales sumados a los SCMs usados en esta investigación, establecieron una demanda excesiva de alcalinidad. Esta circunstancia limitó el aprovechamiento pleno de la reactividad de las adiciones, derivada de aspectos como la composición química y mineralógica, distribución de tamaño de partículas, área superficial y cantidad de fase amorfa.

- Para este estudio resultó desfavorable la adición de SCMs al cemento base en términos de protección del acero a la corrosión, detectándose claramente su efecto perjudicial en el comportamiento electroquímico de las probetas de concreto. La influencia negativa de la sustitución parcial de cemento comercial (CPC) por adición consistió en la reducción del $\mathrm{pH}$ de la pasta de cementante de los concretos debido a la mayor penetrabilidad del dióxido de carbono. Esta carbonatación fue acentuada por la reacción puzolánica disminuida originada en el exceso de adición.

\section{REFERENCIAS}

Ahmaruzzaman, M. (2010). A review on the utilization of fly ash. Prog. Energ. Combust. 36 (3), 327-363. http://dx.doi. org/10.1016/j.pecs.2009.11.003.

ACI 201.2R-01 (2000). Guía para la Durabilidad del Hormigón. Informe técnico ACI Committee 201.

ACI 233R-95 (2000). Ground Granulated Blast-Furnace Slag as a Cementitious Constituent in Concrete, Technical Report (Reapproved 2000). ACI Committee 233.

ACI 232.2R-03 (2003). Use of Fly Ash in Concrete, Technical Report ACI Committee 232.

ACI 222.R-01 (2010). Protection of Metals in Concrete Against Corrosion, Technical Report (Reapproved 2010). ACI Committee 232. 
ASTM A262 (2002). Standard Practices for Detecting Susceptibility to Intergranular Attack in Austenitic Stainless Steels, ASTM International, West Conshohocken, Pa.

ASTM A276 (2010). Standard Specification for Stainless Steel Bars and Shapes, ASTM International, West Conshohocken, Pa.

ASTM C188 (1995). Standard Test Method for Density of Hydraulic Cement, ASTM International, West Conshohocken, Pa.

ASTM C204 (1996). Standard Test Method for Fineness of Hydraulic Cement by Air Permeability Apparatus, ASTM International, West Conshohocken, Pa.

ASTM C187-98 (1998). Standard Test Method for Normal Consistency of Hydraulic Cement, ASTM International, West Conshohocken, $\mathrm{Pa}$

ASTM C230/C230M (1998). Standard Specification for Flow Table for Use in Tests of Hydraulic Cement, ASTM International, West Conshohocken, $\mathrm{Pa}$.

ASTM C109/C109M (1999). Standard Test Method for Compressive Strength of Hydraulic Cement Mortars (Using 2-in. or [50-mm] Cube Specimens), ASTM International, West Conshohocken, $\mathrm{Pa}$

ASTM C150 (1999). Standard Specification for Portland Cement, ASTM International, West Conshohocken, Pa.

ASTM C191 (1999). Standard Test Method for Time of Setting of Hydraulic Cement by Vicat Needle, ASTM International, West Conshohocken, Pa.

ASTM C989 (1999). Standard Specification for Ground Granulated Blast-Furnace Slag for Use in Concrete and Mortars, ASTM International, West Conshohocken, $\mathrm{Pa}$

ASTM C192/C192M (2000). Standard Practice for Making and Curing Concrete Test Specimens in the Laboratory, ASTM International, West Conshohocken, $\mathrm{Pa}$

ASTM C311 (2000). Standard Test Methods for Sampling and Testing Fly Ash or Natural Pozzolans for Use as a Mineral Admixture in Portland-Cement Concrete, ASTM International, West Conshohocken, $\mathrm{Pa}$.

ASTM C595 (2000). Standard Specification for Blended Hydraulic Cements, ASTM International, West Conshohocken, Pa.

ASTM C39/C39M (2001). Standard Test Method for Compressive Strength of Cylindrical Concrete Specimens. ASTM International, West Conshohocken, $\mathrm{Pa}$.

ASTM C618 (2005). Standard Specification for Coal Fly Ash and Raw or Calcined Natural Pozzolan for Use in Concrete, ASTM International, West Conshohocken, Pa.

ASTM C1157/C1157M (2011). Standard Performance Specification for Hydraulic Cement, ASTM International, West Conshohocken, Pa. http://dx.doi.org/10.1520/C1157_ C1157M-11.

ASTM D3173 (2011). Standard Test Method for Moisture in the Analysis Sample of Coal and Coke, ASTM International, West Conshohocken, Pa. http://dx.doi. org/10.1520/D3173-11.

ASTM G102-89 (1999). Standard Practice for Calculation of Corrosion Rates and Related Information from Electrochemical Measurements, Reapproved 1999, ASTM International, West Conshohocken, Pa.

ASTM G59-97 (2003). Standard Test Method for Conducting Potentiodynamic Polarization Resistance Measurements. Reapproved 2003. ASTM International, West Conshohocken, $\mathrm{Pa}$.

Bermúdez-Odriozola, M.A. (2007). Corrosión de las armaduras del hormigón armado en ambiente marino: Zona de carrera de mareas y zona sumergida. Tesis doctoral, Universidad Politécnica de Madrid - Escuela Técnica Superior de Ingenieros de Caminos, Canales y Puertos, Madrid, España.

Bostanci, S.C., Limbachiya, M., Kew, H. (2015). Portland slag and composites cement concretes: engineering and durability properties. J. Clean Prod. Article in press, 1-11. http:// dx.doi.org/10.1016/j.jclepro.2015.08.070.

BS EN 196-9 (2003). Methods of testing cement, Heat of hydration, Semi-adiabatic method, British Standard.

Dong, B., Qiu, Q., Xiang, J., Huang, C., Sun, H., Xing, F., Liu, W. (2015). Electrochemical impedance interpretation of the carbonation behavior for fly ash-slag-cement materials. Constr. Build. Mater. 93, 933-942. http://dx.doi. org/10.1016/j.conbuildmat.2015.05.066.

Fernández-Carrasco, L., Torréns-Martín, D., Martínez-Ramírez, S. (2012). Carbonation of ternary building cementing materials.
Cement Concrete Comp. 34 (10), 1180-1186. http://dx.doi. org/10.1016/j.cemconcomp.2012.06.016.

Flatt, R.J., Roussel, N., Cheeseman, C.R. (2012). Concrete: An eco material that needs to be improved. J. Eur. Ceram. Soc. 32 (11), 2787-2798. http://dx.doi.org/10.1016/j.jeurceramsoc. 2011.11 .012$.

Fonseca, F.S., Godfrey, R.C., Siggard, K. (2015). Compressive strength of masonry grout containing high amounts of class $\mathrm{F}$ fly ash and ground granulated blast furnace slag. Constr. Build. Mater. 94, 719-727. http://dx.doi.org/10.1016/ j.conbuildmat.2015.07.115.

Galán, I. (2011). Carbonatación del hormigón: combinación de $\mathrm{CO}_{2}$ con las fases hidratadas del cemento y frente de cambio de $\mathrm{pH}$. Tesis doctoral. Universidad Complutense de Madrid, Madrid, España.

Jalal, M., Pouladkhan, A., Harandi, O.F., Jafari, D. (2015). Comparative study on effects of Class F fly ash, nano silica and silica fume on properties of high performance self compacting concrete. Constr. Build. Mater. 94, 90-104. http://dx.doi.org/10.1016/j.conbuildmat.2015.07.001

Jeong, Y., Park, H., Jun, Y., Jeong, J.H., Oh, J.E. (2015). Microstructural verification of the strength performance of ternary blended cement systems with high volumes of fly ash and GGBFS. Constr. Build. Mater. 95, 96-107. http://dx.doi. org/10.1016/j.conbuildmat.2015.07.158.

Juenger, M.C.G., Siddique, R. (2015). Recent advances in understanding the role of supplementary cementitious materials in concrete. Cement Concrete Res. 78, 71-80. http://dx.doi. org/10.1016/j.cemconres.2015.03.018.

Lothenbach, B., Scrivener, K.,Hooton, R.D. (2011). Supplementary cementitious materials. Cement Concrete Res. 41, 1244-1256. $\mathrm{http} / / / \mathrm{dx}$.doi.org/10.1016/j.cemconres.2010.12.001.

Makhloufi, Z., Chettih, M., Bederina, M., Hadj Kadri, E.L., Bouhicha, M. (2015). Effect of quaternary cementitious systems containing limestone, blast furnace slag and natural pozzolan on mechanical behavior of limestone mortars. En: Constr. Build. Mater. 95, 647-657. http://dx.doi.org/ 10.1016/j.conbuildmat.2015.07.050

McCarter, W.J., Chrisp, T.M., Starrs, G., Adamson, A., Basheer, P.A., Nanukuttan, S.V., Srinivasan, S., Green, C. (2013). Characterization of physio-chemical processes and hydration kinetics in concretes containing supplementary cementitious materials using electrical property measurements. Cement Concrete Res. 50, 26-33. http://dx.doi.org/10.1016/ j.cemconres.2013.03.008.

NTC 3459 (2001). Agua para la elaboración de concreto, Norma Técnica Colombiana, Icontec Internacional, Bogotá, Colombia.

NTC 121 (2014). Especificación de desempeño para cemento hidráulico, Norma Técnica Colombiana, Icontec Internacional, Bogotá, Colombia.

Özbay, E., Sahmaran, M., Lachemi, M., Yücel, H.E. (2013). Self-Healing of Microcracks in High-Volume Fly-AshIncorporated Engineered Cementitious Composites. $A C I$ Mater. J. 110 (1), 3-43.

Pacheco, F., Miraldo, S., Labrincha, J.A., De Brito, J. (2012). An overview on concrete carbonation in the context of eco-efficient construction: Evaluation, use of SCMs and/ or RAC. Constr. Build. Mater. 36, 141-150. http://dx.doi. org/10.1016/j.conbuildmat.2012.04.066.

Schneider, M., Romer, M., Tschudin, M., Bolio, H. (2011). Sustainable cement production - present and future. Cement Concrete Res. 41, 642-650. http://dx.doi.org/10.1016/j. cemconres.2011.03.019.

Shaikh, F.U.A., Supit, S.W.M. (2015). Compressive strength and durability properties of high volume fly ash (HVFA) concretes containing ultrafine fly ash (UFFA). Constr. Build. Mater. 82, 192-205. http://dx.doi.org/10.1016/j. conbuildmat.2015.02.068.

Song, H., Saraswathy, V. (2006). Studies on the corrosion resistance of reinforced steel in concrete with ground granulated blast-furnace slag - An overview. J. Hazard. Mater. B138, 226-233. http://dx.doi.org/10.1016/j.jhazmat.2006.07.022.

Song, H. Saraswathy, V. (2007) Corrosion Monitoring of Reinforced Concrete Structures - A Review. Int. J. Electrochem. Sci. 2, 1-28. 